\title{
On Demand Multicasting Routing Protocol compared with AODV and FSR in Mobile Ad hoc Network
}

\author{
Kavita Kundlik Bondre ${ }^{1}$, Prof. Ajitsinh.N.Jadhav ${ }^{2}$ \\ ${ }^{1}$ Dept. of Electronics \& Telecommunication Engg D.Y.Patil College of Engg. Tech.K.Bawda \\ Kolhapur, India \\ Email:kkbondre@rediffmail.com \\ ${ }^{2}$ Dept. of Electronics Engg D.Y.Patil College of Engg. \& Tech.K.Bawda Kolhapur, India \\ Email: saisam@indiatimes.com
}

\begin{abstract}
Mobile ad hoc network is a collection of wireless nodes that dynamically organize themselves to form a network without the need for any fixed infrastructure or centralized administration. The network topology dynamically changes frequently in an unpredictable manner since nodes are free to move. Support for multicasting is essential in such environment as it is considered to be an efficient way to deliver information from source nodes to many client nodes. On-Demand Multicast Routing Protocol is a protocol for routing multicast and unicast traffic throughout adhoc wireless mesh networks. ODMRP creates routes on demand so they suffer from a route acquisition delay,although it helps reduce network traffic in general. In future this results will be compared with AODV and FSR protocol and ODMRP performs better as compared with AODV and FSR protocol.
\end{abstract}

Keywords :Mobile adhoc network,routing protocol, ODMRP protocol

\section{Council for Innovative Research}

Peer Review Research Publishing System

Journal: International Journal of Management \& Information Technology

Vol. 6, No. 1

editor@cirworld.com

www.cirworld.com, member.cirworld.com 


\section{INTRODUCTION}

A mobile ad-hoc network is composed of a group of mobile, wireless nodes which cooperate in forwarding packets in multi-hop fashion without any centralized administration. Routing consists of finding a path from source to destination host. Routing is complex in large networks because of many potential intermediate destinations a packet might traverse before reaching its destination [3]. Multicasting has emerged as one of the most focused areas in the field of networking. As the technology and popularity of the Internet have grown, applications that require multicasting (e.g., video conferencing) are becoming more widespread. Another interesting recent development has been the emergence of dynamically reconfigurable wireless ad hoc networks to interconnect mobile users for applications ranging from disaster recovery to distributed collaborative computing. Multicast plays a key role in ad hoc networks because of the notion of teams and the need to show data/images to hold conferences among them.Multicasting is the transmission of data-grams (packets) to a group of zero or more hosts identified by a single destination address. A multicast packet is typically delivered to all members of its destination host group with the same reliability as regular unicast packets. Multicasting reduces the communication cost for applications that sending the same data to many recipients instead of sending via multiple unicast, multicast reduces the channel bandwidth, sender and router processing and delivery delay. Multicasting protocol for the Adhoc network is On-demand Multicast Routing Protocol (ODMRP). The use of multicasting with the network has many benefits. Multicasting reduces the communication cost for applications that sends the same data to many recipients [4].Limited bandwidth, constrained power, and mobility of network hosts make the multicast protocol design particularly challenging. To overcome these limitations, we have developed the On-Demand Multicast Routing Protocol. (ODMRP). ODMRP applies on-demand routing techniques to avoid channel overhead and improve scalability. It uses the concept of forwarding group [5], a set of nodes responsible for forwarding multicast data on shortest paths between any member pairs, to build a forwarding mesh for each multicast group. In view of need to evaluate the performance of ODMRP with other common routing protocols used now days.

\section{RELATED WORK}

\section{Routing Protocols}

There are several routing protocols proposed for wireless ad hoc networks. Classification of routing protocols is as given below:

- Proactive or table-driven routing protocols

- Reactive or on-demand routing protocols.

- Hybrid routing protocols.

Pro-active or Table-Driven routing protocols require each node to maintain up-to-date routing information to every other node (or nodes located within a specific region) in thenetwork. On-demand routing protocols are designed to reduce the overheads in Table-Driven protocols by maintaining information for active routes only as and when required. Hybrid protocols combine the features of both proactive and reactive routing strategies to scale well with the increase in network size and node density. Following protocols are compared in this paper by evaluating the performance of each on the basis of PDR, end to end delay and throughput.

\section{Adhoc On-demand Distance Vector Routing (AODV)}

AODV is a reactive protocol in which the routes are created only when they are needed. It uses traditional routing tables, one entry per destination, and sequence numbers to determine whether routing information is up-to-date and to prevent routing loops. An important feature of AODV is the maintenance of time-based states in each node: a routing entry not recently used is expired. In case of a route is broken the neighbors can be notified. Route discovery is based on query and reply cycles, and route information is stored in all intermediate nodes along the route in the form of route table entries. The following control packets are used: routing request message (RREQ) is broadcasted by a node requiring a route to another node, routing reply message (RREP) is unicasted back to the source of RREQ, and route error message (RERR) is sent to notify other nodes of the loss of the link. HELLO messages are used for detecting and monitoring links to neighbors [10].

\section{3 .Fisheye State Routing (FSR)}

Application layer routing has received little attention in the adhoc domain. An application layer approach has various advantages like routing is easy to deploy. It does not require changes at the network layer [2]. The construction of logical structure hides routing complications such as link failure instances, which are left to be taken care of at routing layer. Application layer routing exploits the capabilities of lower layer protocols in providing reliability, congestion control, flow control or security according to the needs of application. The Fisheye State Routing (FSR) algorithm for ad hoc networks introduces the notion of multi-level "scope" to reduce routing update overhead in large networks. A node stores the link state for every destination in the network. It periodically broadcasts the link state update of a destination to its neighbors with a frequency that depends on the hop distance to that destination (i.e., the "scope" relative to that destination)[11]. FSR is an improvement of GSR. The large size of update messages in GSR wastes a considerable amount of network bandwidth. In FSR, each update message does not contain information about all nodes. Instead, it exchanges information about closer nodes more frequently than it does about farther nodes thus reducing the update message size. So each node gets accurate information about neighbors and the detail and accuracy of information decreases as the distance from node increases. 


\section{PROPOSED WORK}

\section{ODMRP : On Demand Multicast Routing Protocol (ODMRP):}

Features of ODMRP :

- Simplicity

- Low channel and storage overhead

- Usage of Up-to-date shortest routes

- Reliable construction of routes and forwarding group

- Robustness to host mobility

- Maintenance and utilization of multiple paths

- Exploitation of the broadcast nature of the wireless environment

ODMRP is a mesh based rather than conventional tree based scheme and uses a forwarding group concept [4]. The drawbacks in maintaining multicast trees in adhoc network are frequent tree reconfiguration and non-shortest path in a shared tree. In ODMRP, group membership and multicast routes are established by the source on demand when a multicast source has packets to send, but no route to the multicast group, it broadcasts Join-Query control packets to the entire network. This control packet is periodically broadcast to refresh the membership information and updates route.

\section{Basic operation of ODMRP protocol:}

In ODMRP, group membership and multicast routes are established and updated by the source on demand. Similar to ondemand unicast routing protocols, a request phase and a reply phase comprise the protocol (see Fig. 1). While a multicast source has packets to send, it periodically broadcasts to the entire network a member advertising packet, called a JOIN REQUEST.This periodic transmission refreshes the membership information and updates the route as follows. When a node receives a non-duplicate JOIN REQUEST, it stores the upstream node ID (i.e., backward learning) and rebroadcasts the packet.When the JOIN REQUEST packet reaches a multicast receiver, the receiver creates or updates the source entry in its Member Table. While valid entries exist in the Member Table, JOIN TABLES are broadcasted periodically to the neighbors. When a node receives a JOIN TABLE, it checks if the next node ID of one of the entries matches its own ID. If it does, the node realizes that it is on the path to the source and thus is part of the forwarding group. It then sets the FG Flag and broadcasts its own JOIN TABLE built upon matched entries. The JOIN TABLE is thus propagated by each forwarding group member until it reaches the multicast source via the shortest path. This process constructs (or updates) the routes from sources to receivers and builds a mesh of nodes, the forwarding group.

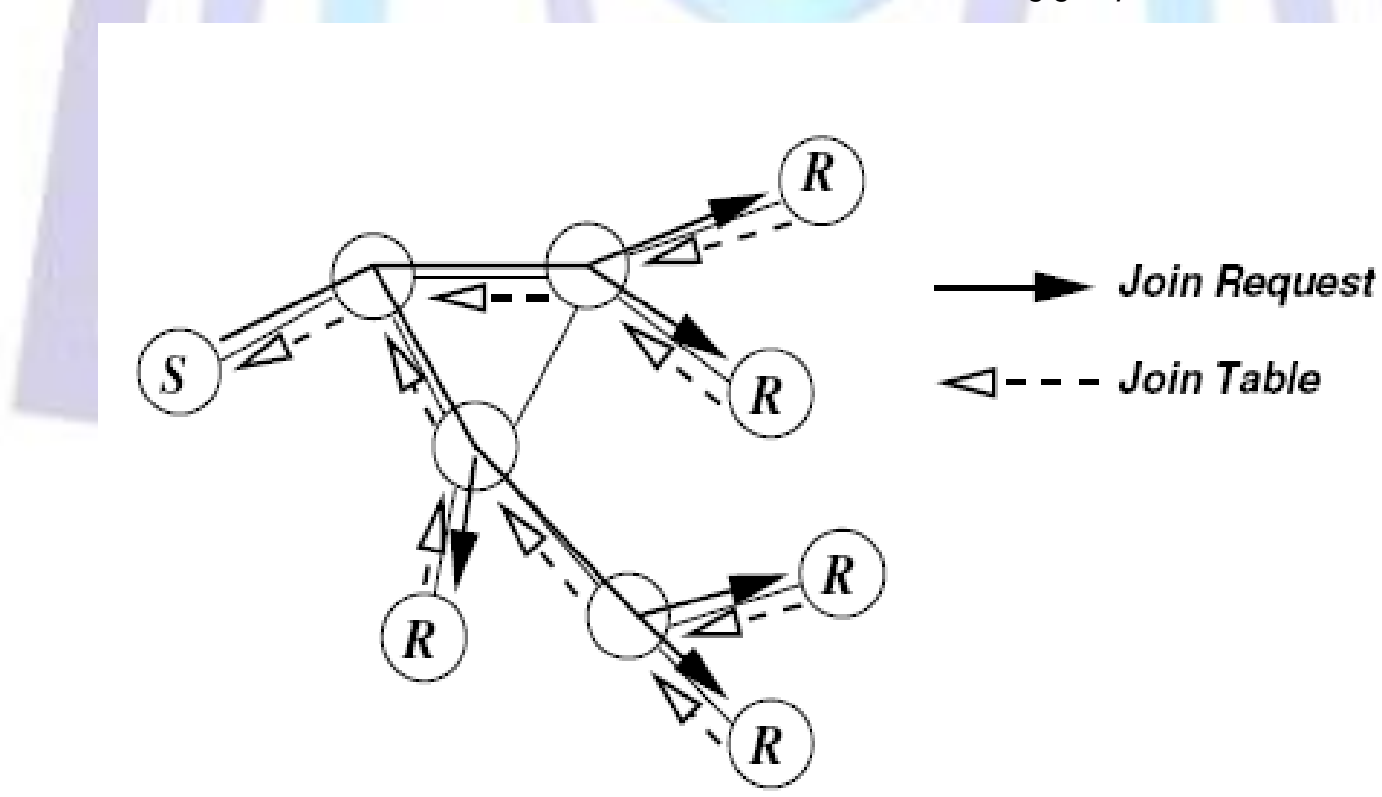

Fig. 1. On-Demand Procedure for Membership Setup and Maintenance

We have visualized the forwarding group concept in Fig. 2. The forwarding group is a set of nodes in charge of forwarding multicast packets. It supports shortest paths between any member pairs. All nodes inside the .bubble. (multicast members and forwarding group nodes) forward multicast data packets. Note that a multicast receiver can also be a forwarding group node if it is on the path between a multicast source and another receiver. The mesh provides richer connectivity among multicast members compared to trees. Flooding redundancy among forwarding group helps overcome node displacements and channel fading. Hence, unlike trees, frequent reconfigurations are not required. 


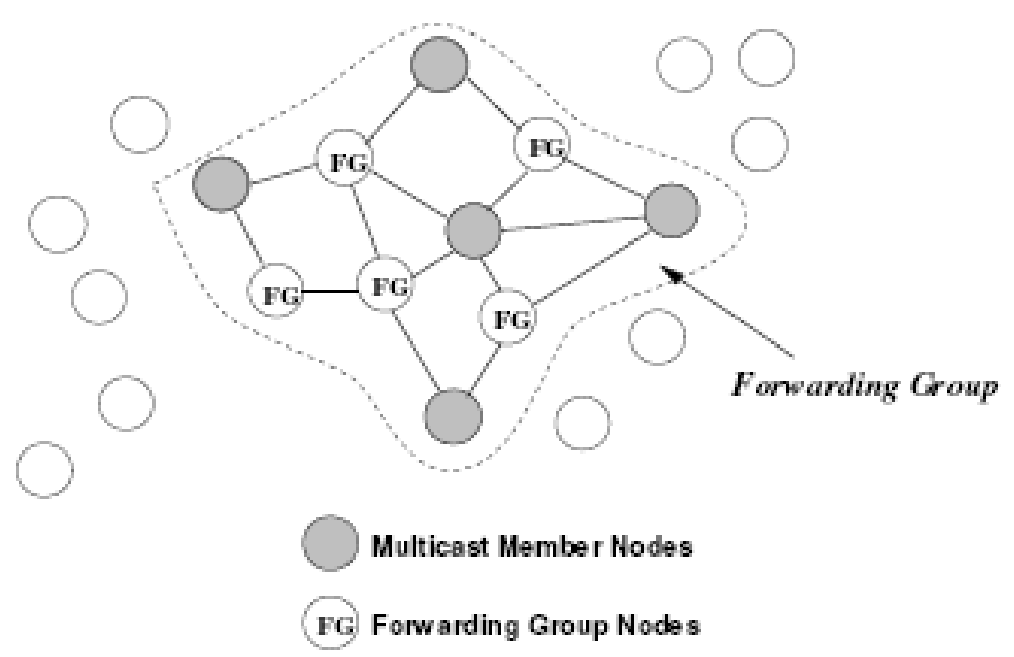

Fig. 2. The Forwarding Group Concept.

The performance metrics used for evaluation are:

- Average Throughput

- Packet delivery ratio

A .Average Throughput

$\times-($ Gnuplot (window id : 0 )

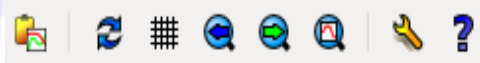

Average throughput Vs number of nodes

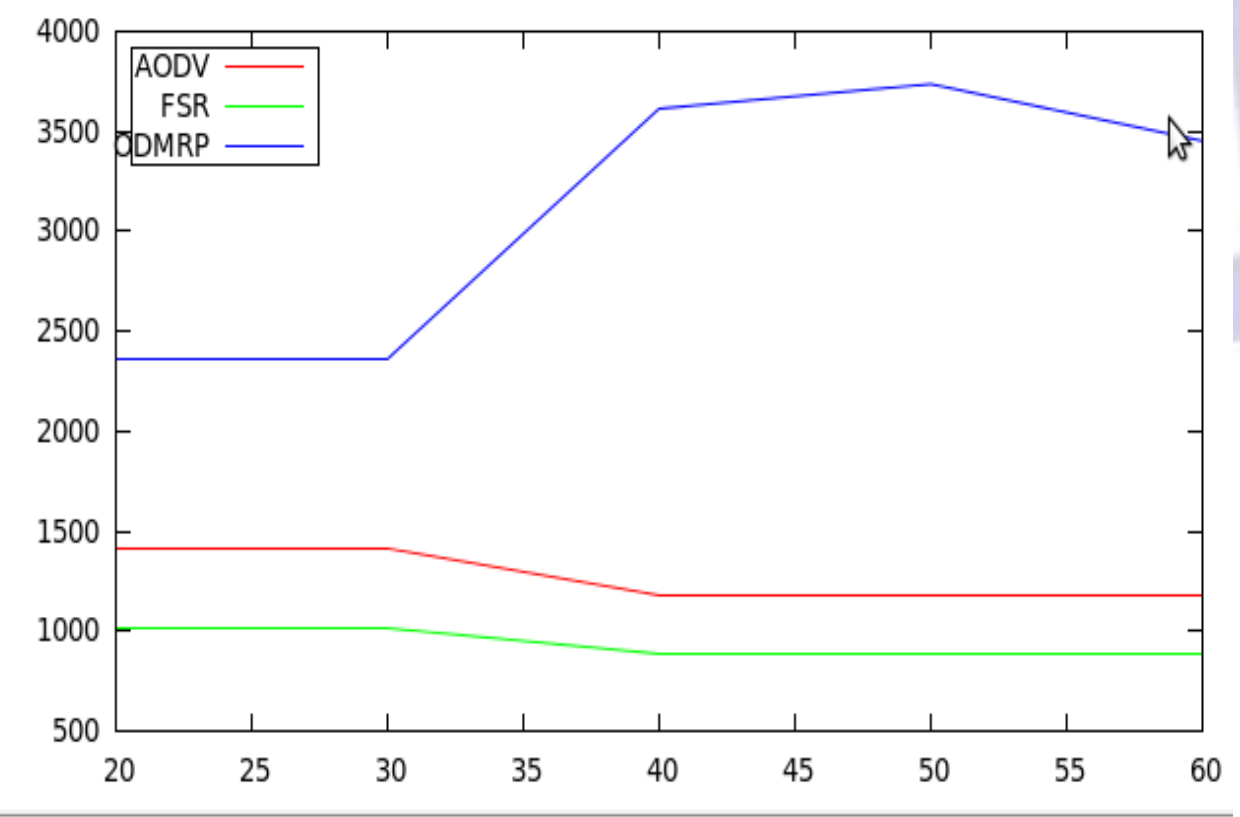

$58.7716,3554.31$

Fig 3: Average throught vs no.of nodes

Throughput indicates rate of communication per unittime. Throughput in these experiments is evaluated for all three routing protocols for varying node mobility and nodes. Figure 1 shows the average throughput (bytes per simulation time 
of $200 \mathrm{sec}$ ) for three protocols with changing number of nodes i.e. for 15, 30, 45 and 60 nodes. Average throughput is $3232.3,1380.80$ and 566.26 bytes per simulation time for ODMRP, AODV and FSR respectively.

B Packet delivery ratio

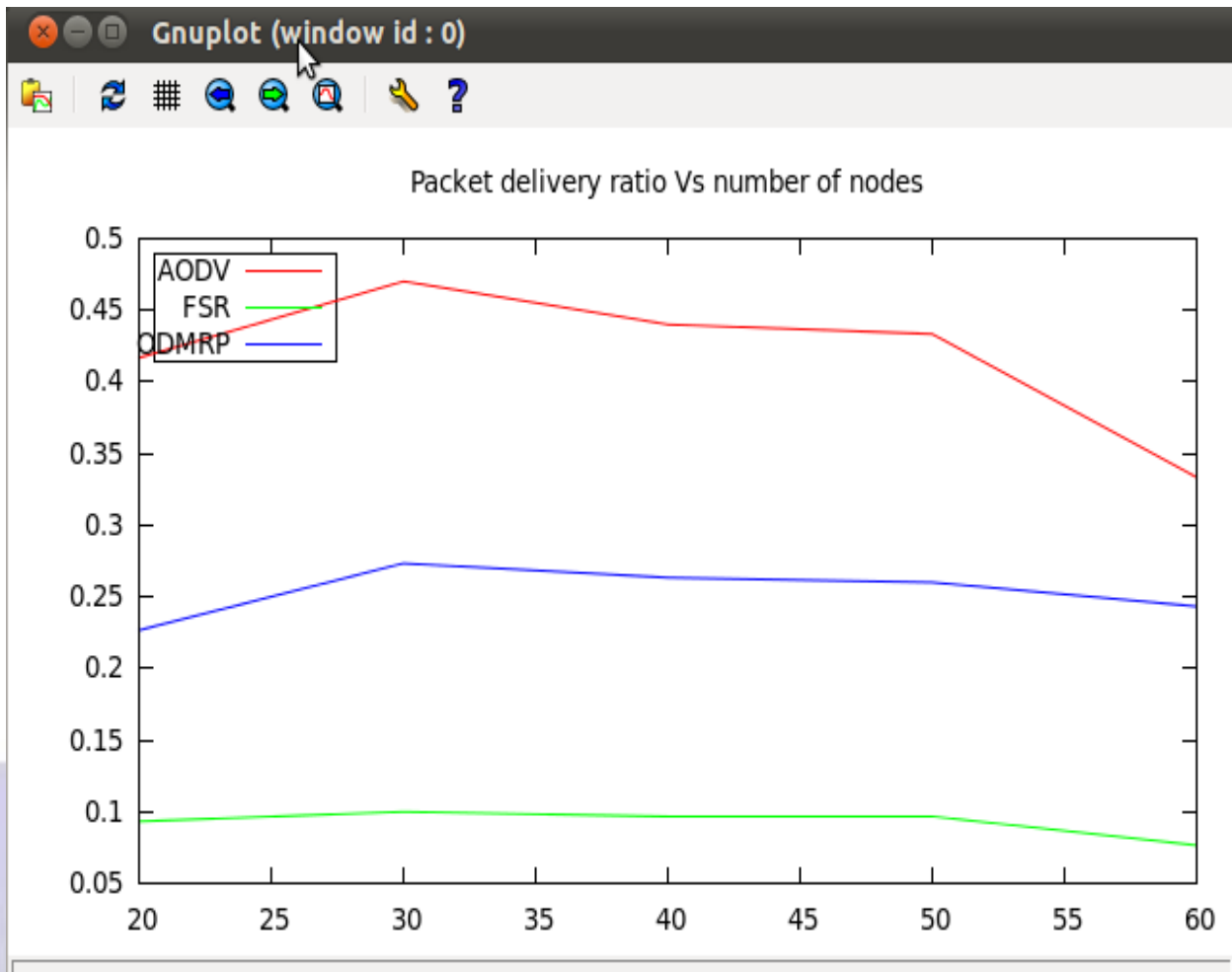

$16.8838,0.480297$

Fig 4: packet delivery vs no .of nodes

Packet Delivery Ratio (PDR) is number of successfully delivered legitimate packets to number of generated legitimate packets.

$$
P D R=\frac{\text { Total number of packets sent }}{\text { Total number of packets received }}
$$

A higher value of PDR indicates that most of the packets are being delivered to the higher layers and is a good indicator of the protocol performance. Average packet delivery ratio for ODMRP is evaluated as 0.4 , for AODV 0.7 and for FSR is 0.3 as shown in figure 3.

\section{CONCLUSION}

In this paper we are evaluated on demand multicasting routing protocol for mobile adhoc network. ODMRP creates routes on demand so they suffer from a route acquisition delay, although it helps reduce network traffic in general . future work will consider the comparison and implementation of ODMRP protocol with AODV protocol and FSR protocol considering with the varying number of nodes In above parameter.

\section{REFERENCES}

[1] R. Mkhija and R.Saluja, "Performance Comparison of Ad-Hoc Routing Protocol In Different Network Size",Proc. of 2nd NationalConf.MathematicalTechniques:Emerging Paradigms in Electronics and IT Industries,(Sept 26-28, 2008).

[2] Elizabeth M. Royer, Santa Barbara and Chai-Keong Toh, "A Review of Current Routing protocols for Ad Hoc Mobile Wireless Networks", IEEE Personal Communications, Vol 6, pp. 46-55 (April 1999).

[3] Sung-Ju Lee, William Su and Mario Gerla, "On-Demand Multicast Routing Protocol (ODMRP) for Ad Hoc Networks", INTERNET-DRAFT <draft-ietf-manetodmrp-02.txt>( November 2002).

[4] Thomas Kunz and Ed Cheng, "On-Demand Multicasting in Ad-Hoc Networks: Comparing AODV and ODMRP", Proc. of the 22nd IEEE International Conf. on Distributed Computing Systems (ICDCS'02), Vol-2, pp 1063-6927 (2002). 
[5] S. Corson, J. Macker, "Mobile ad hoc networking (MANET): Routing protocol performance issues andevaluation considerations", RFC 2501 (1999).

[6] Guangyu Pei, Mario Gerla, Tsu-Wei Chen, "Fisheye State Routing in Mobile Ad Hoc Networks", Proc. Of IEEE ICC'00 (2000).

[7] Shapour Joudi Begdillo, Mehdi Asadi and Abolfazl Toroghi Haghighat, "Improving Packet Delivery Ratio in ODMRP with Route Diversity", Int. Journal of Computer Sc. and Network Security (IJCSNS), Vol. 7,No.12, pp 146-155 (2007). 